\title{
Effects of Dopant Types and Contents on Morphology and Strength Properties of PANI/PAN Electrospun Nanofibers
}

\author{
Taohai Yan ${ }^{1,2,3}$, Jinhua Jiang ${ }^{1}$ and Nanliang Chen $^{1, a}$ \\ ${ }^{1}$ College of Textiles, Donghua University, Shanghai 201620, P.R. China. \\ ${ }^{2}$ Clothing and Design Faculty, Minjiang University, Fuzhou, Fujian 350121, P.R. China \\ ${ }^{3}$ Fujian Key Laboratory of Novel Functional Fibers and Materials, Minjiang University, Fuzhou Fujian 350121, P.R. \\ China
}

\begin{abstract}
In this paper, the electrospinning of polyaniline under different dopant conditions were studied, the experimental phenomena was introduced, analyzed the relationship between the nanofiber surface morphology of DBSA-PANI/PAN, HCl-PANI/PAN and PTSA-PANI/PAN membranes and dopant type and content. The average diameter of DBSA-PANI/PAN electrospinning fiber membrane showed a decreasing trend when the content of doped polyaniline increased $1 \%-4 \%$, and reached a minimum value of $780 \mathrm{~nm}$ when the concentration was $4 \%$. The strength of DBSA-PANI/PAN fiber membrane was the highest when the concentration of doped polyaniline was $1 \%$, and the maximum intensity was $1.8 \times 105$ $\mathrm{Pa}$. The average diameter of $\mathrm{HCl} / \mathrm{PANI} / \mathrm{PAN}$ fiber membranes decreases with the increase of doped polyaniline concentration, and it fluctuates up and down within a small range. When the content of doped polyaniline was increased to $4 \%$, the average fiber diameter reached a minimum of $420 \mathrm{~nm}$. The strength of the HCl-PANI/PAN fiber membrane was the highest when the concentration of doped polyaniline was $4 \%$, and the maximum intensity was $1.08 \times 10$ $5 \mathrm{~Pa}$. The average diameter of the PTSA-PANI/PAN electrospun fiber membrane was the smallest at $2 \%$ doped polyaniline content and was $430 \mathrm{~nm}$, the strength of PTSA-PANI/PAN fiber membrane is the highest when the content of doped polyaniline is $2 \%$, and the maximum intensity is $3.9 \times 104 \mathrm{~Pa}$. In this paper, the basic research of doped polyaniline electrospinning nanofibers has made a useful supplement to the existing research of polyaniline nanofibers, and has a reference value for the application of polyaniline.
\end{abstract}

\section{Introduction}

In 1862, polyaniline was first used as a dyestuff in H.Letheby's research. There have been three phases of polyaniline research: the discuss on "Aniline Black" as a dyestuff, the research of organic semiconductor, and as well as extensive studies of conducting polymer[1]. Due to the lack of profound theoretical knowledge of macromolecular at that time, the further study of polyaniline had been dragged on for nearly a century before 1960s. In 1976 MacDiarmid found that polyaniline boasted unique properties such as electric conduction. This led to polyaniline being considered a conductive polymer with widespread applications. Polyaniline is easily obtainable as a raw material, is easily manipulated by synthetic processes, has a high conductivity, a unique doping performance, an excellent electromagnetic microwave absorbing performance, is environmental stabile, chemically stabile, and has optical properties[2]. Efficient doping of the macromolecular functional protic acids

${ }^{\mathrm{a} C}$ orresponding author. Email: nlch@dhu.edu.cn 
with PANI molecular chains, such as dodecylbenzenesulfonic acid(DBSA), camphorsulphonic acid(CSA), paratoluenesulfonic acid (TSA), poly (acrylic acid)(PAA), Sodium pyrophosphate(TSPP), naphthalenesulfonic acid (NSA) have been performed. The acid acts as a template or steric stabilizer to form a doped PANI limb dispersion. Previous studies have found that functional proton sulfonic acids that contain aromatic rings and 10 to 15 carbon chain alkyl groups are excellent dopants for PANI. The dodecyl benzene sulfonic acid (DBSA) is the most commonly doped macromolecule, which could effectively improve the dispersibility of the product and does not weaken the performance of polyaniline [3]. Electrospinning is a process that uses electrostatic forces to form fibers[4]. This process uses a high voltage supply, which induces the charge of a certain polarity into a polymer solution or a polymer melt that is accelerated to a collector that has the opposite polarity. The obtained electrospun fibers have a small diameter, in the micrometer to nanometer range[5]. The polymer content of the electrospun polyaniline composite fiber influences the fiber morphology by changing the content of the PANI or the polymer to make a stable jet during electrospinning, which forms a stable fiber. The diameter and the electrical conductivity of the polyaniline composite fibers have increased and the mechanical properties have been enhanced under specific conditions [6].

Five groups of doped polyaniline solutions with different mass fractions were designed, $1 \mathrm{wt} . \%, 2$ wt. $\%, 3$ wt. $\%$, 4 wt. $\%, 5$ wt. $\%$, PAN content in solution remains at $10 \mathrm{wt} . \%$, the molar masses of the three dopants are the same as those of polyaniline, the three dopants are p-Toluenesulfonic acid (PTSA), Dodecyl benzene sulfonic acid (DBSA), hydrochloric acid(HCL), the relationship between nano-composite fiber membrane morphology and dopant species was analyzed.

\section{MATERIALS AND METHODS}

\subsection{Materials and equipment}

Polyaniline (PANI), p-Toluenesulfonic acid (PTSA) and polyacrylonitrile (PAN) (MW 150,000) was purchased from Beijing Kuol Chemical Technology Co., Ltd.. (Beijing, China). Dodecyl benzene sulfonic acid (DBSA) was purchased from Zhanfan Chemical co., LTD. (Guangzhou, China). Hydrochloric acid was purchased from Damao Chemical Reagent Factory (Tianjin, China). N,N-Dimethylformamide (DMF) was purchased from Tianjin Zhiyuan Chemical Reagent Co., Ltd (Tianjin, China). All chemicals were analytical reagent and were used without alteration. The microcomputer type conductivity meter DDBJ-350 was purchased from Qiwei instrument co., LTD. (Hangzhou, China). The high pure water system EXPLORER was purchased from Chenhuang Scientific Instrument co., LTD (Shanghai, China). The high voltage DC power supply DW-P503-1ACDF was purchased from Dongwen High Voltage Power Supply co., LTD (Tianjin, China). The two-channel trace injection pump WZS-50F6 was purchased from Smith Medical Instrument co., LTD (Zhejiang, China).

\subsection{Methods}

Electrospinning PAN, $6 \%, 10 \%$, and $12 \%$ pure PAN nanofilms, it was found that $10 \%$ PAN nanomembranes had the best spinning effect, then add $1 \%, 2 \%, 3 \%, 4 \%, 5 \%$ equimolar mass of polyaniline and dopant in 10\% PAN solution in DMF. The solution was first dispersed in an ultrasonic cleaning apparatus for 10 minutes, and then the solution was returned to a magnetic stirrer and stirred for about 12 hours to obtain a uniform and stable solution. The doped polyaniline and PAN solution was put into a needle. The distance between the metal needle and the receiving plate was $10 \mathrm{~cm}$, the voltage was $15.00 \mathrm{kv}$, and the pump flow rate was $1 \mathrm{ml} / \mathrm{h}$.

\subsection{Morphological characterization of doped-PANI/PAN electrospun fibers membrane}

A scanning electron microscope (Hitachi S-4800) was used to quantify the morphology and the 
surface of the electrospun fibers. The samples were sputtered with gold and pictures were taken. The diameters of more than 50 electrospun fibers were measured via scanning electron microscopy (SEM) images using Image $\mathrm{J}$ software. The average diameters and standard deviations were calculated.

\subsection{Fiber membrane strength test}

The equipment used for this fiber membrane strength test was a 3365-INSTRON strength tester. For each pattern, take 6 sets of rectangular specimens of $1 \mathrm{~cm} \times 10 \mathrm{~cm} \times 0.1 \mathrm{~mm}$ to obtain fiber membrane strength data, calculate the average fiber strength based on the formula.

Calculate the average fiber strength according to the formula:

$$
\sigma=\mathrm{P} \div \mathrm{A}
$$

In the formula: $\sigma$-Breaking strength or volumetric strength $(\mathrm{Pa}), P-$ Breaking force $(\mathrm{N})$, $A$ - Cross section area (m2).

\section{Results and Discussion}

\subsection{Electrospun PAN nanofiber membrane}

\subsubsection{SEM analysis of PAN electrospun nanofiber membrane}

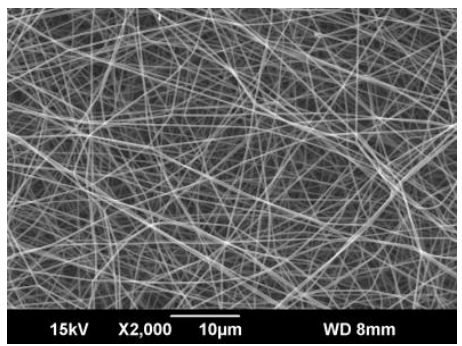

(a) $8 \%$ PAN

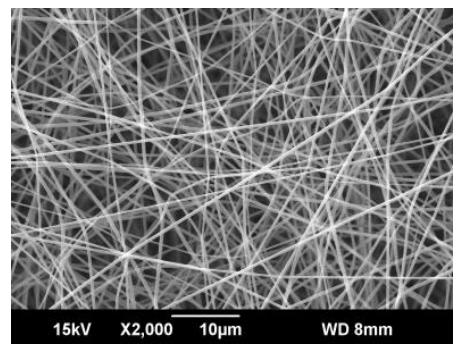

(b) $10 \%$ PAN

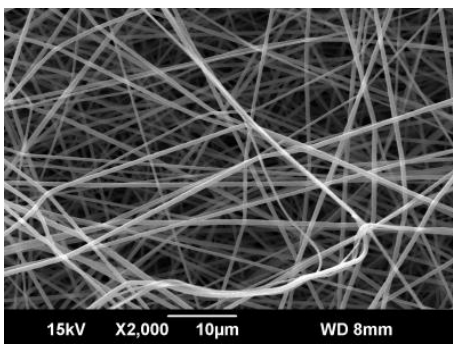

(c) $12 \%$ PAN

Figure 1. SEM of polyacrylonitrile nanofiber membranes with different concentrations

Figure 1 shows the morphological structure of the nanofiber membranes under different concentrations of PAN spinning solution obtained after 2,000-fold magnification. The fibers of the three nanofiber membranes are uniform and smooth. The diameters of nanofibers with concentrations of $8 \%, 10 \%$, and $12 \%$ were $121 \mathrm{~nm}, 173 \mathrm{~nm}$, and $299 \mathrm{~nm}$ respectively. It is easy to see that the fiber diameter increases with the increase of PAN concentration.

\subsection{DBSA-PANI/PAN NANOFIBER MEMBRANE}

\subsubsection{SEM analysis of DBSA-PANI/PAN nanofiber membrane}




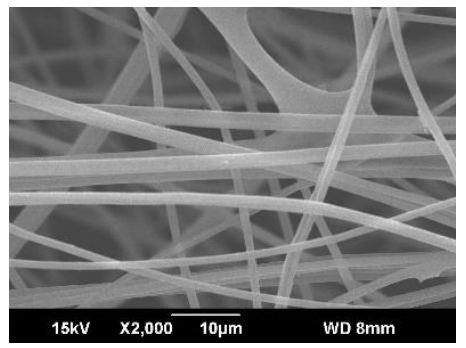

(a)PAN10\%+PANI-DBSA1\%

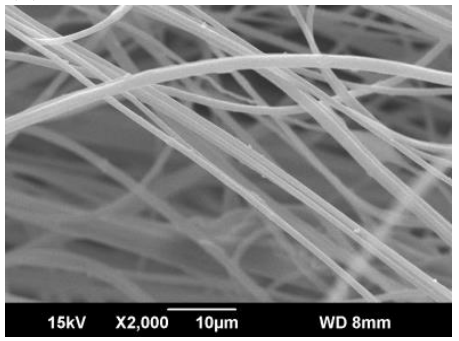

(d)PAN10\%+PANI-DBSA4\%
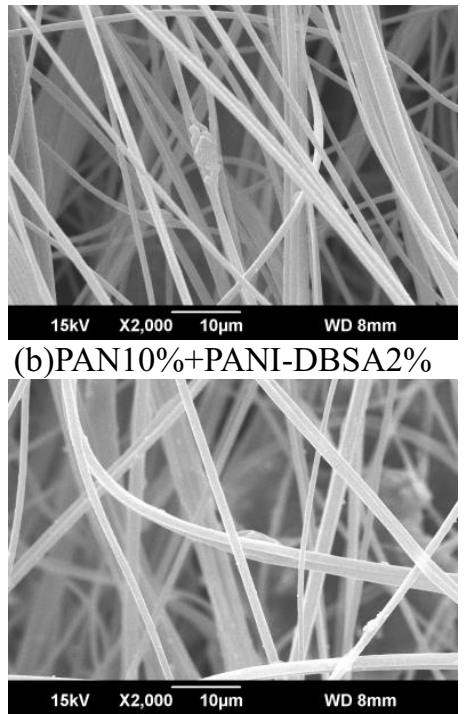

(e) PAN10\%+PANI-DBSA $\%$

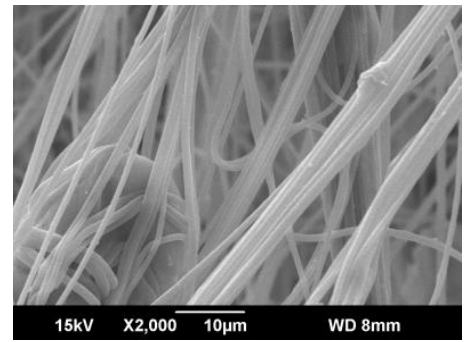

(c)PAN10\%+PANI-DBSA3\%

Figure 2. SEM of fiber membranes at different concentrations of PANI-DBSA

In Figure 2(a), (b), (c), (d) and (e) represent SEM of 1\%, 2\%, 3\%, 4\%, and 5\% DBSA-PANI electrospun membranes, the average fiber diameters are $910 \mathrm{~nm}$ and $890 \mathrm{~nm}, 830 \mathrm{~nm}, 780 \mathrm{~nm}$, and $880 \mathrm{~nm}$ respectively. Electrospun nanofibers have a relatively smooth dry phase and a uniform fiber diameter. With the increase of polyaniline and dodecyl benzene sulfonic acid concentration, the average fiber shows a tendency to decrease, the average diameter of the fiber reaches a minimum value when the concentration of polyaniline and dodecyl benzene sulfonic acid increases to $4 \%$. And the concentration reaches $5 \%$, the mean diameter of the fiber increases and the average diameter and concentration of the fiber are almost the same as the average diameter at $2 \%$.

\subsubsection{Strength test of DBSA-PANI/PAN nanofiber membrane}

Strength of $1 \%, 2 \%, 3 \%, 4 \%$, and $5 \%$ DBSA-PANI electrospun membranes are $2.12 \times 10^{5} \mathrm{~Pa}$, $1.42 \times 10^{5} \mathrm{~Pa}, 7.4 \times 10^{4} \mathrm{~Pa}, 1.8 \times 10^{5} \mathrm{~Pa}$ and $6.1 \times 10^{4} \mathrm{~Pa}$ respectively. As the content of doped polyaniline increases, the fiber strength gradually decreases, but at a concentration of $4 \%$, the strength reaches a peak and then decreases. Its strong value rule is not very obvious and has a certain degree of randomness.

\subsection{HCL-PANI/PAN NANOFIBER MEMBRANE}

\subsubsection{SEM analysis of HCI-PANI/PAN nanofiber membrane}

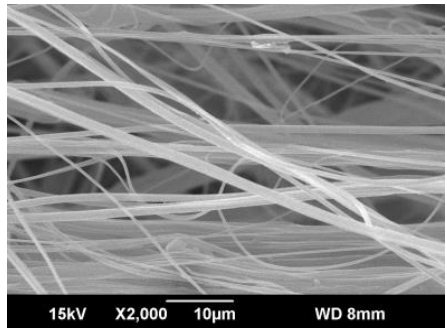

(a)PAN10\%+HCl-PANI1\%

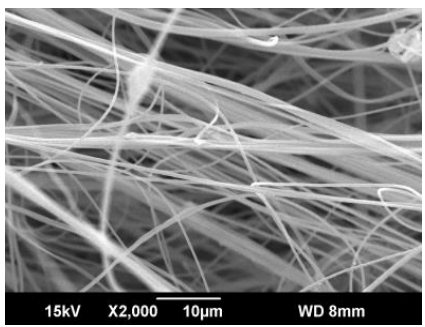

(b)PAN10\%+HCl-PANI 2\%

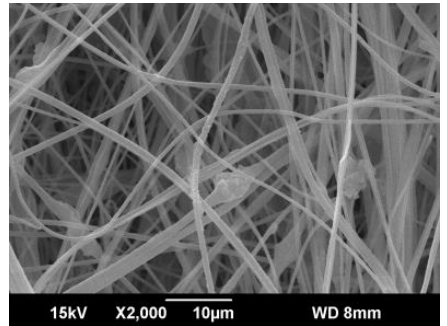

(c)PAN10\%+HCl-PANI 3\% 


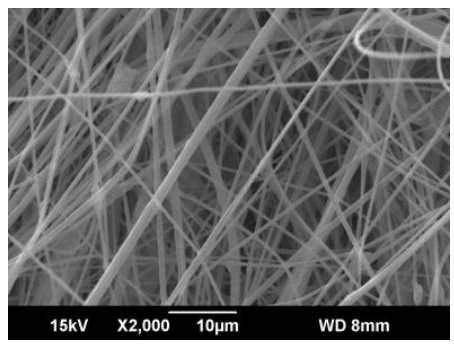

(d)PAN10\%+HCl-PANI 4\%

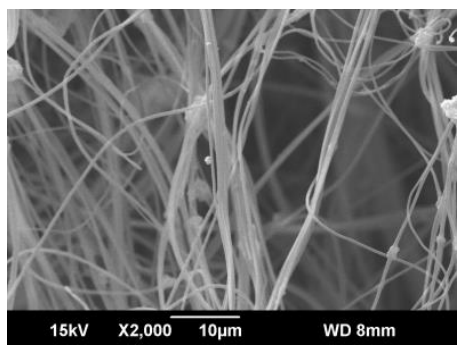

(e)PAN10\%+HCl-PANI 5\%

Figure 3 SEM of fiber membranes at different HCl-PANI concentrations

In Figure 3(a), (b), (c), (d) and (e) represent SEM of 1\%, 2\%, 3\%, 4\%, and 5\% DBSA-PANI electrospun membranes, the average fiber diameters are $550 \mathrm{~nm}, 450 \mathrm{~nm}, 480 \mathrm{~nm}, 420 \mathrm{~nm}$ and $610 \mathrm{~nm}$ respectively. The fiber diameter is uniform, the strips are clear, there are no obvious impurities, and the film formation is relatively good. With the increase of HCl-PANI concentration, the diameter of nanofiber membranes showed a decreasing trend overall and fluctuate up and down within a small range. When the concentration of polyaniline and hydrochloric acid was increased to $4 \%$, the average diameter of the fibers reached a minimum. And when the concentration reached $5 \%$, the average diameter of the fibers rapidly increased.

\subsubsection{Strength test of HCI-PANI/PAN nanofiber membrane}

Strength of $1 \%, 2 \%, 3 \%, 4 \%$, and $5 \%$ HCl-PANI electrospun membranes are $6.6 \times 10^{4} \mathrm{~Pa}, 5.7 \times 10^{4} \mathrm{~Pa}$, $6.3 \times 10^{4} \mathrm{~Pa}, 1.08 \times 10^{5} \mathrm{~Pa}$ and $5.3 \times 10^{4} \mathrm{P}$ respectively. As the content of doped polyaniline increases, the fiber strength gradually decreases, but at a concentration of $4 \%$, the strength reaches a peak and then decreases. Its strong value rule is not very obvious and has a certain degree of randomness. With the increase of the concentration, the strength of the fibrous membrane showed a small fluctuation at first and then decreased. When the concentration increased to $4 \%$, the strength of the fibrous membrane reached a maximum, and then the strength of the fibrous membrane showed a downward trend. Because that the solution concentration increases polyaniline content in the composite fiber system increases, the fiber membrane strength increased significantly, but increased to a certain concentration, the solubility of solute in the solution decreased, the fiber membrane strength instead showed a downward trend.

\subsection{PTSA-PANI/PAN NANOFIBER MEMBRANE}

\subsubsection{SEM analysis of PTSA -PANI/PAN nanofiber membrane}

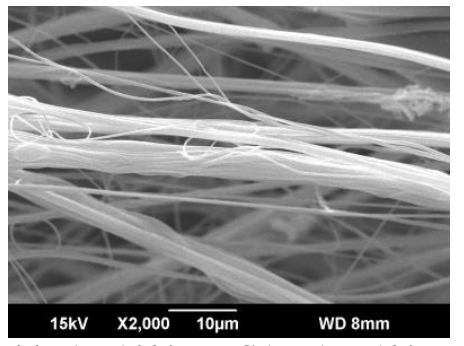

(a)PAN $10 \%+$ PTSA-PANI $1 \%$

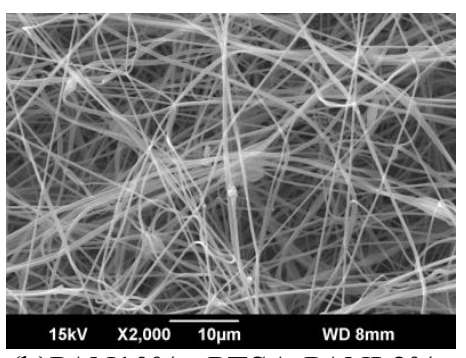

(b)PAN10\%+ PTSA-PANI $\%$

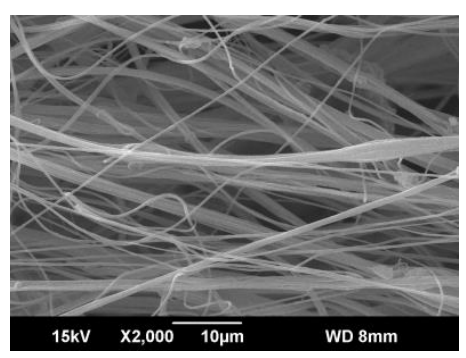

(c)PAN10\%+ PTSA-PANI 3\%

Figure 4. SEM of fiber membrane at different PTSA-PANI concentrations

In Figure 4(a), (b) and (c) represent SEM of 1\%, 2\% and 3\% PTSA-PANI electrospun membranes, the average fiber diameters are $580 \mathrm{~nm}, 430 \mathrm{~nm}$ and $530 \mathrm{~nm}$ respectively. With the increase of the 
concentration of doped polyaniline, the average fiber diameter showed a trend of decreasing first and then increasing. There was obvious adhesion between the fibers, and a small amount of spindle-shaped spherical particles appeared. With the increase of polyaniline concentration, the viscosity of the solution increased, and the adhesion was more obvious.

\subsubsection{Strength test of PTSA-PANI/PAN nanofiber membrane}

Strength of $1 \%, 2 \%, 3 \%$ and $4 \%$ PTSA-PANI electrospun membranes are $2.4 \times 10^{4} \mathrm{~Pa}, 3.9 \times 10^{4} \mathrm{~Pa}$, $1.1 \times 10^{4} \mathrm{~Pa}$ and $2.1 \times 10^{4} \mathrm{~Pa}$ respectively. With the increase of the concentration of doped polyaniline, the strength of the fiber membrane showed a trend of increasing first and then decreasing. At a concentration of $2 \%$, the strength of the fiber membrane reached a maximum value, and the strength of the fiber membrane decreased afterwards.

\section{Conclusion}

Under the same conditions, the mean diameters of the fibrous membranes of $\mathrm{HCl} / \mathrm{PANI} / \mathrm{PAN}$ and PTSA/PANI/PAN were similar and the smallest, and the average diameter of the DBSA/PANI/PAN membrane was the largest. Under the same conditions, the fiber membrane strength of DBSA/PANI/PAN was the highest, the fiber membrane strength of $\mathrm{HCl} / \mathrm{PANI} / \mathrm{PAN}$ was the second, and the strength of PTSA/PANI/PAN fiber membrane was small. Hydrochloric acid as an inorganic small molecule protonic acid, its polyaniline-doped solubility is low, the small molecule acid is easily dedoped, resulting in poor mechanical properties of the doped polyaniline fiber membrane. Dodecyl benzene sulfonic acid has a large molecular mass and a large size, the binding capacity of the molecular chain is improved after doping, so that the final fiber membrane exhibits a higher strength, resulting in a higher strength of the final fibrous membrane. The nanostructured polyaniline fiber has unique physical and chemical properties. When the size of the polyaniline material is at the nanometer level, due to its structural advantages, it has a larger size, while it has a smaller size effect and a tunneling effect than the conventional fiber. The application prospect of doped polyaniline nanofiber film is broad.

\section{References}

1. Jung, Y., Baeg, K. J., Kim, D. Y., Someya, T., Park, S. Y.. Synth. Met., 159 (2009)

2. Engin, F. Z., Usta, İ. J. Text. I., 106(2015)

3. Lakshmidevi, V., Yelamaggad, C. V., Venkataraman, A.. ChemistrySelect, 3 (2018)

4. Dural-Erem, A., Erem, H. H., Ozcan, G., Skrifvars, M.. J. Text. I., 106(2015)

5. Nurwaha, D., Han, W., Wang, X.. J. Text. I., 104(2013)

6. Sujith, K., Asha, A. M., Anjali, P., Sivakumar, N., Subramanian, K. R. V., Nair, S. V., Balakrishnan, A.. Mater. Lett. , 67(2012) 